\title{
Topografía alegórica de la exclusión del otro: Tierra adentro de Angelina Muñiz Huberman
}

\author{
Allegorical Topography of the Exclusion of the Other: \\ Inland by Angelina Muñiz Huberman
}

\author{
E. Helena Houvenaghel \\ ORCID: 0000-0002-7877-2065 \\ Universiteit Utrecht, ICON, Países Bajos \\ e.m.h.houvenaghel@uu.nl
}

FLORIEN SERLET

FWO-Vlaanderen, Universiteit Gent, Bélgica

florien.serlet@gmail.com

Resumen:

La obra de la autora judío-mexicana Angelina Muñiz-Huberman (Francia, 1936) destaca por dos aportes: una de índole temática, la transmisión de la cultura y del pensamiento judíos, y otra de índole formal, la escritura "transgenérica" a través de la fusión de los géneros literarios. ¿Cómo se relacionan entre sí dichos aportes? Una de las obras tempranas de Angelina Muñiz-Huberman, Tierra adentro (1977), parece apuntar a un proceso de refuerzo mutuo en el cual el modo alegórico desempeña un papel fundamental. Tierra adentro, en el nivel literal del texto, narra la trayectoria de un sefardí expulsado de la España del Siglo de Oro a la Tierra Santa y evoca, simultáneamente, en el segundo nivel de lectura, la persecución del pueblo asquenazi en el siglo XX. La alegoría propicia una reflexión ética sobre el carácter repetitivo del mismo patrón de la exclusión de la 
comunidad judía a través de la historia y simultáneamente estimula la fusión de los géneros literarios tradicionales.

Palabras clave:

Angelina Muñiz-Huberman, escritura transgenérica, alegoría, ética, cultura judía, exclusión del otro.

Abstract:

The work of Jewish-Mexican writer Angelina Muñiz-Huberman (France, 1936) stands out for two contributions: one of a thematic nature, namely the transmission of Jewish culture and thought, and the other of a formal nature, namely 'transgeneric' writing via the fusion of literary genres. How do these contributions relate to each other? One of Muñiz-Huberman's early works, Tierra adentro (Inland, 1977), seems to point out toward a process of mutual reinforcement in which the allegory plays a key role. Tierra adentro, on the literal level, narrates how a Sephardic Jew is expulsed from the Spain of the Golden Age and makes his way to Holy Land. On the secondary level, it simultaneously evokes the persecution of the Askhenazi Jews in the 20th century. The allegorical mode encourages an ethical reflection on the repetitive character of the pattern of expulsion and persecution of the Jewish community throughout history, and simultaneously stimulates the fusion of the traditional literary genres.

Keywords:

Angelina Muñiz-Huberman, transgeneric writing, allegory, ethics, Jewish culture, exclusion of the other.

DOI: https://doi.org/10.36798/critlit.v0i23.372

Recibido: 17 de diciembre de 2020

Aceptado: 14 de abril de 2021 


\section{La alegoría para instruir deleitando}

La obra literaria de la autora judío-mexicana Angelina Muñiz-Huberman ${ }^{1}$ (Francia, 1936) destaca por dos aportes: una de índole temática, la difusión de la cultura, la historia y el pensamiento judíos, y otra de índole formal, la escritura "transgenérica"2 a través de la fusión de los géneros literarios. Desde el punto de vista temático, la cultura y el pensamiento judíos permean la obra de Muñiz-Huberman. Desde el punto de vista formal, Angelina Muñiz-Huberman fusiona en su práctica escritural prosa filosófica, ensayo histórico, ficción narrativa y poesía en un conjunto, un "todo" literario (Houvenaghel 2020). ${ }^{3}$ ¿Cómo se relacionan entre sí dichos aportes? Una de las obras tempranas de Angelina Muñiz-Huberman, Tierra aden-

${ }^{1}$ Angelina Muñiz-Huberman es hija de padres españoles que, al estallar la Guerra Civil se trasladaron a Francia, donde la autora nació. Tras pasar por Cuba, la familia se naturalizó en México en 1942. Se formó en la Universidad Nacional Autónoma de México y en la New York City University. Es profesora de literatura comparada en la Universidad Nacional Autónoma de México. Es autora de 45 libros, ganadora de múltiples premios literarios, entre los cuales destacan el Premio Sor Juana Inés de la Cruz (1993) y el Premio Nacional de Artes y Literatura 2018 de México.

2 El prefijo "trans" se caracteriza por un "movimiento inherente" que "sobrepasa cualquier idea de límite estático y supone una superación de la dialéctica abierto-cerrado, dentro-afuera, realidad-ficción" (González de Canales 203-4). En combinación con el adjetivo "genérico", el prefijo produce un efecto dinamizador y liberador al apuntar "hacia una nueva perspectiva, menos rígida y más permeable, que permite una aproximación polidireccional al campo genérico y evita así su encasillamiento" (González de Canales 2014).

3 La crítica menciona a menudo la escritura transgenérica de Angelina Muñiz-Huberman, pero profundiza poco en el significado de dicha escritura. En este sentido ha sido pionero el número monográfico Escribir en Nepantla, la prosa sin fronteras de Angelina Muñiz. Huberman (Houvenaghel 2015). Posteriormente, la práctica de la escritura transgenérica se puso en relación con el inconformismo de la escritora en el número monográfico Angelina Muñiz Huberman: Una voz inconformista (Houvenaghel y Carrillo 2021) y se estudió la relación entre el misticismo de Angelina Muñiz Huberman y su escritura transgenérica (Houvenaghel 2020). 
tro (1977), parece apuntar a un proceso de refuerzo mutuo en el cual el modo alegórico desempeña un papel fundamental.

Tierra adentro forma parte de la primera trilogía de Angelina Muñiz-Huberman que reflexiona sobre, sucesivamente, tres conceptos: "la guerra" (La Guerra del Unicornio 1983), "el exilio" (Morada Interior 1972) y "la exclusión del otro" (Tierra adentro 1977). ${ }^{4}$ En la primera parte de la trilogía, La Guerra del Unicornio, la autora propone una aproximación cabalística a "la Guerra" y considera la lucha entre lo bueno y lo malo como una etapa necesaria en la trayectoria hacia la armonía (Houvenaghel, "Using the wisdom..."). La segunda parte de la trilogía, Morada interior, profundiza, desde un ángulo místico, en el exilio espiritual del alma y su búsqueda de la unión con lo divino. En la tercera parte de la trilogía, Tierra adentro, Muñiz Huberman tematiza, desde un punto de vista histórico-ético, la expulsión y persecución de la comunidad judía y la búsqueda de un refugio seguro del pueblo judío. ${ }^{5}$

La primera parte de la trilogía, La Guerra del Unicornio, hace uso del modo alegórico. Una primera capa literal del libro refiere a un conflicto armado situado en un contexto fantástico mientras que el significado secundario de la obra refiere a la Guerra de España (1936-1939). El mensaje ético transmitido por La Guerra del Unicornio se relaciona con la visión cabalística de la lucha entre lo bueno y lo malo (Houvenaghel, "Using the wisdom"). Acorde con nuestra hipótesis, la última parte de la trilogía, Tierra adentro, también se construye sobre la base de la alegoría.

${ }^{4} \mathrm{El}$ orden de las partes de la trilogía es engañoso por los problemas de publicación que la escritora tuvo. Angelina Muñiz-Huberman (cit. en Salazar y Rodríguez Plaza 168) explica que La Guerra del Unicornio, que se publicó como último volumen de la trilogía, debería haber sido la primera parte de la trilogía.

${ }^{5}$ El título Tierra adentro se relaciona, acorde con la autora, precisamente con este territorio propio, con la tierra soñada, con un anhelo de llegar a una patria, una tierra que sea suya (Gambarte 28-29). En este contexto de una tierra soñada, conviene apuntar que la traducción al inglés de la novela se titula Dreaming of Safed. Safed es un lugar en el norte de Israel en el cual, en el siglo XVI, se construyó un centro de la mística en el que la mística judía y la mística musulmana se estudian juntas. 
La interpretación alegórica de textos tiene una larga tradición en el campo de la religión y filosofía. Al aplicar el modo alegórico en su escritura, Angelina Muñiz-Huberman no solo conecta, de manera transgenérica, con textos de índole religiosa y filosófica, sino que también guía al lector hacia una lectura entre líneas de sus libros. La escritura de textos alegóricos, cuya misión consiste en instruir deleitando (Bloom 164), nace efectivamente, con una intención didáctica (Fletcher 30):

En esencia, entonces, alegoría ... es un modo literario que presenta conscientemente en un mismo tiempo al menos dos significados: uno puede ser designado como primario, o como un significado superficial literal. El otro puede ser designado como secundario, o como un significado abstracto, es decir, uno con penetrante moralista o didáctica intención. (Bloom 164; traducción mía)

Ahora bien, de manera correspondiente, Tierra adentro transmite dos significados al mismo tiempo. Se trata, en el nivel literal, de una narración de ficción neohistórica (Menton 42-46) que relata cómo el personaje de Rafael, un joven judío español expulsado de la España del Siglo de Oro, emprende el viaje dificultoso a Jerusalén para iniciar, en este territorio de refugio, una nueva vida con su pareja. ${ }^{6}$ La crítica se ha centrado en profundizar en la riqueza y complejidad de esta primera capa del texto al destacar de manera muy acertada la impronta de la tradición picaresca (Friedman, Mateo Gambarte, Sanz Villanueva, Zamudio) y del género del Bildungsroman (Payne) en Tierra adentro.

Este primer nivel literal de lectura se puede leer como una alegoría de la expulsión del pueblo sefardí de España. Sin embargo, proponemos que se puede agregar, además, una segunda capa de significado relacionado con la expulsión y persecución del pueblo

${ }^{6}$ Los protagonistas se llaman Rafael y Miriam, nombres de los hijos de Muñiz-Huberman, a quienes dedica el libro Tierra adentro. 
asquenazi por el régimen nazi. Las dos capas de Tierra adentro sobreponen dos casos emblemáticos de la exclusión de la comunidad judía, ocurridos en espacios y tiempos muy diferentes.

\section{La primacía del orden espacial en Tierra adentro}

Para acceder a la segunda capa interpretativa, proponemos una relectura de Tierra adentro en clave espacial. El protagonismo dado a la trayectoria de la huida del protagonista Rafael y la importancia concedida a la descripción de los espacios de su itinerario nos permiten aproximar Tierra adentro al género de la novela de viaje (Albuquerque García, "El 'relato de viajes"” y "Los libros"). ${ }^{7}$ En este género, "la modalidad descriptiva se impone a la narrativa" (Albuquerque García, "El 'relato de viajes"” 16-17): "el discurso se represa en la travesía, en los lugares, en todo lo circundante (personas, situaciones, costumbres, leyendas, mitos, etc.) que se convierten en el nervio mismo del relato". El espacio crea, efectivamente, en Tierra adentro, la estructura de la obra, a través de referencias y descripciones de los lugares recorridos por el judío Rafael en su trayectoria de huida que le lleva de España a Israel.

A esta predominancia del espacio como elemento estructurante se opone, en Tierra adentro, la vaguedad del marco temporal. Tierra adentro se inicia con un solo dato temporal concreto, la fecha de nacimiento del protagonista sefaradí: "Mi nombre es Rafael. Nací

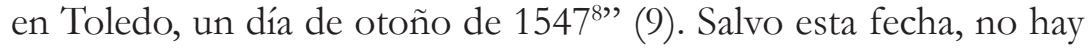
más indicaciones temporales exactas que permitan situar los acontecimientos siguientes ni precisar el tiempo que transcurre durante la narración (Menton 159). En el texto sí se hallan referencias vagas

7 Albuquerque García ("El 'relato de viajes” 16, 21) distingue en la categoría de "libro de viajes" entre "relatos de viajes" (de modalidad factual, que linda con la historia) y "novelas de viajes" (de modalidad ficcional, literaria).

${ }^{8}$ Año de nacimiento de Miguel de Cervantes. 
a elementos históricos — tales como la inquisición y las guerras religiosas - y referencias literarias —a la novela picaresca, los dramas de honor y la literatura mística- cuyo efecto consiste en relacionar la narración con el siglo de Oro. Con todo, el marco temporal de Tierra adentro se caracteriza, pues, por la escasez de precisiones temporales y, de ahí, por cierta vaguedad y ambigüedad. Esta vaguedad del marco temporal es la que nos conduce a desplazar el acento hacia el espacio y el movimiento espacial.

El mecanismo subyacente a la interpretación alegórica de Tierra adentro es, precisamente, (el movimiento en) el espacio. La alegoría coteja el movimiento en el espacio (la trayectoria de la huida del judío sefardí de España a Jerusalén) y el movimiento en el tiempo (la historia de la Shoah que lleva a la creación del estado de Israel). El agente alegórico es Rafael: se establece un paralelismo entre la trayectoria ficticia de dicho personaje sefardí en el siglo XVI y la historia de la expulsión y huida del pueblo asquenazi en las décadas de 1930 y 1940. La concordancia entre espacio y tiempo, y de ahí entre viaje e historia, es una estrategia a la que se apunta, a nuestro parecer, en el texto mismo. Así, por ejemplo, la siguiente reflexión de Rafael sugiere la relación estrecha e incluso el carácter intercambiable de las dos dimensiones: "Después de este descanso, otra vez emprendemos la marcha. Empiezo a sentir . . cómo el espacio se vuelve tiempo. Cada palmo recorrido es tiempo ..." (156).

El segundo nivel de lectura de Tierra adentro se construye, pues, sobre la interconexión cronológica, asegurada por la trayectoria de la huida del judío sefardí Rafael en cuanto hilo conductor, de espacios simbólicamente connotados con determinados acontecimientos o períodos históricos significativos para el pueblo asquenazi, empezando por la exclusión de este por parte del régimen nazi y terminando por la creación del estado de Israel. La descripción de los espacios que Rafael transita linda con la poesía: está dotada de un fuerte valor simbólico y pone énfasis en la expresión de las emociones que los lugares evocan. La descripción no aspira en ningún momento al historicismo ni al realismo, sino que se trata más bien de una descripción poética, sugestiva y portadora de otro significado más allá de lo literal. 
Argumentamos que la interpretación simbólica de la descripción del espacio transcurrido y la ausencia de un marco temporal específico forman la doble base que posibilita la interpretación alegórica del viaje de Rafael.

\section{3. "El espacio se vuelve tiempo"}

El primer espacio recorrido por Rafael que posibilita una correspondencia directa con un episodio clave de la historia del pueblo asquenazi es España, país que es asociado con la expulsión y el terror. El ambiente español se describe como amenazante y oscuro, temible y represivo, dominado por el régimen de terror de la Inquisición. Las numerosas alusiones, por ejemplo, a las torturas o a las quemas de los judíos predominan en la descripción del escenario español. Las columnas de humo y de fuego se convierten en una imagen poderosa y repetitiva (Muñiz-Huberman, Tierra adentro 54, 55, 59, 138): "Mañana habrá una quema de herejes .... La columna de humo, recta, hacia arriba . . . . la columna de fuego se eleva al cielo" (54).

La represión y el rechazo predominan en los recuerdos de la tierra española. Rafael expresa sus sentimientos al despedirse de ella: "Después de todo, nada queda atrás. Si vuelvo la cabeza y veo estos palmos de tierra de España que se me escapan, no hay nada que me traiga alegría” (128). Desde el punto de vista de Rafael, en España no hay futuro para él: hay "por todos lados la desolación, tierra estéril"' (121).

La salida de Rafael de su España natal equivale, en el nivel literal, a la intolerancia de la España del Siglo de Oro, un momento de la historia española quedará siempre marcada, en la historia del pueblo sefardí, como un momento emblemático de rechazo y de represión. Planteamos que el espacio español representa también, de manera alegórica, el ambiente de discriminación y persecución de los judíos asquenazi en los años 30 bajo el primer nazismo en Europa.

El segundo espacio por el que viaja Rafael es Francia. Se insiste, de manera poética, en que ciertas tensiones van aumentándose gra- 
dualmente y que parece que lo peor está por venir: "El aire ya no es tan puro, hay una tensión que empezamos a percibir todos, como el ambiente antes de que estalle la tormenta" (136), "el odio se extiende; los gestos son amenazadores; los ojos, crueles; las palabras, de muerte" (137). Se puede interpretar esta descripción como una referencia al clima de creciente exclusión y a las medidas tomadas en detrimento de los judíos asquenazi en la década de 1930.

El tercer espacio transitado por Rafael con el que podemos establecer una correspondencia directa es Alemania. El propio Rafael observa que las tensiones aumentan gradualmente a medida que va acercándose a Alemania, con lo cual se vincula explícitamente la violencia progresiva con el avance geográfico hacia la tierra en la que Hitler toma el poder: "Ya cerca de la frontera con el reino de Alemania ... vemos de cerca el horror de la guerra" (140). De esta manera, varias señales textuales permiten interpretar el triste pasaje de Rafael a través de tierras alemanas como una de las etapas más horrorosas de la historia del pueblo judío: la Alemania nazi y la Shoah. Así, Tierra adentro describe las guerras en Alemania como las más sangrientas de todas y describe la actitud de los soldados alemanes como la más cruel y absurda, sanguinaria, violenta sin sentido:

Los vencedores no notan la pasividad, siguen acometiendo con la misma furia enloquecida. Solo paran en seco cuando notan la facilidad con que la muerte va segando vidas, pero, recobrados, vuelven al mismo furor desatado y rematan a los que aún quedan con leves muestras de palpitar. Y aún se ensañan con los cadáveres y los siguen destrozando grotescamente, absurdamente. (139)

Cuando se describe una familia entera muerta en Alemania, en una casa "con chimenea humeante . . . un verdadero espejo de la muerte" (Muñiz-Huberman, Tierra adentro 271), se evoca la imagen de uno de los episodios más negros de la historia del siglo XX.

El escenario alemán se describe en términos de tensión, terror y crueldad, a medida que el narrador da cuenta de las destrucciones y masacres provocadas por los soldados alemanes. Alemania se 
relaciona, en suma, con "la locura de la guerra y la muerte" (149). Añádase a eso que, además de la guerra, rige la peste en las tierras de Alemania, lo que da lugar a otras escenas espeluznantes: "Nadie entierra los cadáveres. Los soldados huyen. Los buitres danzan su ronda de muerte. Los chacales y las hienas se regocijan de la estupidez del hombre" (150).

Siguiendo el mismo razonamiento, establecemos un vínculo entre las tierras de Italia, el espacio transitado después de Alemania, y la liberación de Europa del régimen nazi. El mismo texto nos ofrece las claves de lectura que posibilitan dicha interpretación. El pasaje por Italia se describe en términos de luz, de sol, de nuevos horizontes y nuevas esperanzas, subrayando el contraste con la situación durante el régimen nazi:

Entramos en tierras de Italia con un cielo limpio y un sol nuevo. El reloj de la plazuela dice: Post tenebras lux. Y es así, atrás quedó el mundo de las tinieblas, de la muerte, del olvido; hacia adelante es la esperanza, es la tierra de leche y miel ... (158)

Las luchas y guerras violentas de los años cuarenta contrastan diametralmente con la situación en Italia, país en el que se sitúa el puerto del que Rafael podrá salir en barco rumbo a la Tierra Prometida. Italia es un espacio donde hay "luz por todas partes, en los rostros y en las flores", "donde no hay nada oculto, donde todo brilla" (Muñiz-Huberman, Tierra adentro 154). Italia simboliza el final de la época de la oscura Segunda Guerra Mundial y a la vez el inicio de una nueva era iluminada de esperanza. Esta descripción de la tierra de Italia invita a una lectura simbólica que constituye un espacio de paz y tranquilidad después de tanta violencia, sufrimiento, crueldad y muerte, un espacio que da esperanza y posibilidades para construir un nuevo futuro.

El último espacio de la trayectoria de huida del sefardí Rafael es la Tierra Prometida. La descripción del destino final, Jerusalén, es altamente simbólica. "Empieza a sentir lo que es ser feliz: estas casas blancas, no tener miedo. Después de todo, era muy sencillo" 
(173). Con la llegada a la Tierra Prometida, se cierra el círculo para Rafael, un círculo abierto desde la expulsión de su ciudad natal. Al llegar, dice: "He llegado. He tomado un puñado de tierra y la he besado, y la tierra se ha humedecido con mis lágrimas. Es tierra mía, de mi pueblo, de Abraham, de David, de Eliezer Ben Yair, de Simón Bar Kochba. Es tierra mía. Mía. Si quiero, ya puedo morir. . . A Aquí . . nacerán mis hijos y los hijos de mis hijos" (170-1).

Así, proponemos que la llegada de Rafael a Jerusalén puede interpretarse, alegóricamente, como la fundación de una nación soberana para la comunidad judía, el Estado de Israel. O sea, así como la llegada de Rafael a su destino constituye el punto culminante y final de su viaje, la creación de un propio estado judío constituye el clímax de la historia de la comunidad judía. La creación del estado de Israel (1948) fue acompañada de una gran ola de inmigración de los pueblos judíos dispersos por el mundo que encuentran su casa en el país.

En resumen, proponemos que la huida del joven sefardí Rafael en busca de un refugio seguro puede leerse, en clave alegórica, como la persecución del pueblo asquenazi bajo el régimen nazi hasta la creación del Estado de Israel, con Jerusalén como capital, en 1948, y la vuelta de muchos judíos de la diáspora a este territorio de refugio.

\section{Alegoría, escritura transgenérica y ética}

Tierra adentro representa la exclusión y persecución del otro como fenómenos repetitivos y pertenecientes a todas las épocas y a todos los espacios. Esta visión coincide con la reflexión que Muñiz-Huberman lleva a cabo en el ensayo histórico El siglo del desencanto (1999) en torno al conflicto armado, la persecución y la destrucción del otro, considerándolos como fenómenos que persisten a lo largo de la historia de la humanidad. Tierra adentro insiste, por medio del modo alegórico, en los peligros de la continua repetición del mismo (equivocado) patrón. La escritura transgenérica puesta en práctica por la autora promueve la actitud contraria: la autora pone énfasis en la necesidad de cambiar los patrones conocidos, de transformar 
e innovar los géneros literarios, fusionando diferentes formas de prosa con la poesía y rompiendo, así, con la repetición de los viejos esquemas literarios fijos. En este sentido, la forma de Tierra adentro nace de una práctica de escritura inclusiva y complementa el mensaje ético del libro.

La relectura de Tierra adentro en clave alegórica ilustra el papel estimulante que desempeña la alegoría en el proceso de la fusión de los géneros literarios. $\mathrm{Al}$ incorporar diferentes capas de lectura, las cuales remiten a diferentes géneros literarios, en una sola obra, Tierra adentro parece confirmar que la alegoría, "por ser impura, anómala y monstruosa” (Kelley 12; traducción mía), estimula la formación de tipos textuales alternativos ya que "sus diferencias sociales, culturales y estéticas interfieren con la repetición de fórmulas tradicionales" (Kelley 12; traducción mía). Así es que la alegoría ocupa un lugar central en el campo de tensión entre las normas impuestas por los géneros tradicionales y la innovación genérica.

Finalmente, conservar o practicar la alegoría para transmitir un mensaje ético también se puede entender como una manera de reunir de manera transgenérica filosofía, religión, historia e imaginación. Los ataques contra la ficción, emitidos por Platón, declaran que la ficción desvía, por medio de sus frivolidades, la atención del lector de los temas filosóficos y serios que deberían preocuparle. El texto alegórico, sin embargo, oculta por medio de la imaginación las mismas verdades que propone enseñar y la decodificación del texto convierte al lector en un participante activo en la construcción del significado del texto. En la lectura de la alegoría, la búsqueda de la verdad y el placer de dejarse llevar por una historia ficticia van de la mano.

\section{Bibliografía}

Abrams, Meyer Howard. A Glossary of Literary Terms. Holt-Rinehart and Winston, 1988.

Albuquerque García, Luis. "El 'Relato de viajes': hito y formas en la evolución del género". Revista de Literatura, vol. 73, no. 145, junio 2011, pp. 15-34. 
. "Los libros de viaje como género literario". Diez estudios sobre literatura de viajes, editado por Manuel Lucena Giraldo, y Juan Pimentel, CSIC, 2006.

Bloom, Edward. "The Allegorical Principle". A Journal of English Literary History, vol. 18, no. 3, 1951, pp. 163-190.

Fletcher, Angus. Alegoría: Teoría de un modo simbólico. Ediciones Akal, 2002. Friedman, Edward. "Angelina Muñiz's Tierra adentro: (Re)creating the subject". Tradition and Innovation: Reflections on Latin America Jewish Writing, State University of New York, 1993, pp. 179-192. Gambarte, Eduardo Mateo. "Angelina Muñiz-Huberman: Escritora hispano-mexicana". Cuadernos de Investigación Filológica, tomo XVIII, fascículos 1 y 2, 1992, pp. 65-83.

Gambarte, Eduardo Mateo. "Las variadas caras del exilio exploradas por Angelina Muñiz-Huberman”. Homenaje a Angelina Muñi-Huberman, editado por Lilian von der Walde Moheno y Mariel Reinoso Ingliso, Grupo Destiempos, 2014, pp. 26-71.

González de Canales Carcereny, Júlia. "La obra literaria de Enrique Vila-Matas: escritor transnacional por transgenérico e intertextual". Transnacionalidad e hibridez en el ensayo hispánico, Brill, 2017, pp. 201-215.

Houvenaghel, E. Helena. "La hibridez genérica en el espejo: Angelina Muñiz Huberman y María Zambrano". Cuadernos del Hipogrifo. Revista de Literatura Hispanoamericana y Comparada, no. 13, 2020, pp. 12-24.

. "Using the wisdom of Kabbalah to make sense of the Spanish War: Angelina Muñiz-Huberman's War of the Unicorn (1983)". Jewish Imaginaries of the Spanish Civil War: In Search of Poetic Justice, editado por Cynthia Gabbay, Bloomsbury, 2021, en imprenta.

Houvenaghel, E. Helena, coordinadora. Anales de Literatura Hispanoamericana, vol. 44, dossier: Escribir en Nepantla. La prosa sin fronteras de Angelina Muñiz-Huberman, 2015.

Houvenaghel, E. Helena y María Carrillo Espinosa, coordinadoras. Dossier: Angelina Muñiz-Huberman, una voz inconformista. Inti. Revista de literatura bispánica, vol. 1, no. 93, 2021.

Kelley, Therea. Reinventing Allegory. Cambridge University, 1997. 
Menton, Seymour. La nueva novela bistórica de la América Latina, 1979. 1992, Fondo de Cultura Económica, 1993.

Muñiz-Huberman, Angelina. De cuerpo entero. Eljuego de escribir. Universidad Nacional Autónoma de México / Corunda, 1992. . El siglo del desencanto. Fondo de Cultura Económica, 1999. . La guerra del unicornio. Artífice, 1983.

. "La niña en el balcón: una historia en dos partes". Enlace judío, 7 junio 2017.

. Morada interior. Joaquín Mortiz, 1972.

. Tierra adentro. Joaquín Mortiz, 1977.

Payne, Judith. "Writing and Reconciling Exile: The Novels of Angelina Muñiz-Huberman.” Bulletin of Hispanic Studies, vol. 74, no. 4, 1997, pp. 431-459.

Sanz Villanueva, Santos. "Angelina Muñiz: novelista de la generación hispanomexicana”. Dicenda. Cuadernos de Filología Hispánica, no. 2, 1983.

Salazar, Severino y Joaquina Rodríguez Plaza. "Conversación con Angelina Muñiz-Huberman”. Tema y variaciones de literatura 2, coordinado por Antonio Marquet, 1993, pp.163-181.

Zamudio, Luz Elena. "Angelina Muñiz-Huberman, la dualidad asumida”. Homenaje a Angelina Muñi-Huberman, editado por Lilian von der Walde Moheno y Mariel Reinoso Ingliso, Grupo Destiempos, 2014, pp. 79-102. 